\title{
ANALYSIS OF ANECHOIC CHAMBER TESTING OF THE HURRICANE
}

\section{IMAGING RADIOMETER}

\author{
David Fenigstein ${ }^{1}$, Chris Ruf $^{1}$, Mark James $^{2}$, David Simmons ${ }^{2}$, Timothy Miller ${ }^{2}$, Courtney Buckley ${ }^{2}$ \\ ${ }^{1}$ University of Michigan \\ 2455 Hayward St. \\ ${ }^{2}$ NASA/Marshall Space Flight Center \\ 320 Sparkman Drive
}

Ann Arbor, MI 48109-2143

dfenig@umich.edu
Huntsville, AL 35805

\begin{abstract}
The Hurricane Imaging Radiometer System (HIRAD) is a new airborne passive microwave remote sensor developed to observe hurricanes. HIRAD incorporates synthetic thinned array radiometry technology, which use Fourier synthesis to reconstruct images from an array of correlated antenna elements. The HIRAD system response to a point emitter has been measured in an anechoic chamber. With this data, a Fourier inversion image reconstruction algorithm has been developed. Performance analysis of the apparatus is presented, along with an overview of the image reconstruction algorithm.
\end{abstract}

Keywords-microwave radiometer, synthetic aperture radiometry, image reconstruction

\section{INTRODUCTION}

The Hurricane Imaging Radiometer System (HIRAD) is a passive microwave synthetic thinned aperture radiometer that operates at the C-Band frequencies 4, 5, 6 and 6.6 GHz. HIRAD was developed for the purpose of observing wind speed and rain rate in tropical cyclones from aircraft over a wider swath than was possible using available technology. Accurate characterization of aperture synthesis radiometers requires the measurement in an anechoic chamber of antenna-pair interference patterns. This paper describes the procedures and analysis of HIRAD anechoic chamber tests made in October 2009, to verify proper measurement of the antenna-pair interference patterns and to develop an image reconstruction algorithm.

\section{INSTRUMENT DESIGN}

The HIRAD array antenna is composed of linear arrays of stacked multi-resonant radiators, operating at 4, 5, 6, and 6.6 $\mathrm{GHz}[1,2]$. The optimal configuration for 10 1-D antenna elements assigns 36 non-redundant baselines [3]. The elements are spaced at integer multiples of 0.90 inches, resulting in baselines at spacings given in Table I. Each antenna element is connected to a receiver that filters, amplifies and demodulates the signal. The signal is downconverted to an IF frequency and digitized, then introduced to a complex correlator, where each signal is decomposed into in-phase (real) and quadrature-phase (imaginary) parts. The signals are then split into 16 subbands. Complex correlations for all possible pairings of the 10 receivers (45 total) are calculated using complex multipliers at common subbands, giving a measurement of the raw visibility samples [2]. Calibration of the samples is achieved through hot and cold calibration loads internal to each receiver, and a correlated noise diode[2]. This configuration is depicted in the block diagram in Fig. 1.

TABLE I:

HIRAD DESIGN PARAMETERS

\begin{tabular}{|c|c|c|c|c|}
\hline Freq. (GHz) & 4.0, & 5.0 & 6.0 & 6.6 \\
\hline $\begin{array}{c}\text { Antenna spacings (in } \\
\text { units of } \lambda \text { ) }\end{array}$ & .305 & .381 & .457 & .503 \\
\hline IF Bandwidth & \multicolumn{3}{|c|}{$75 \mathrm{MHz}$} \\
\hline IF Subbands & \multicolumn{3}{|c|}{16} \\
\hline \# Array Elements & \multicolumn{3}{|c|}{10} \\
\hline \# Antenna Pairs & \multicolumn{3}{|c|}{45} \\
\hline \# Unique Baselines & \multicolumn{3}{|c|}{$150 \mathrm{MS} / \mathrm{s}$} \\
\hline Sampling Frequency & \multicolumn{3}{c}{} \\
\hline
\end{tabular}




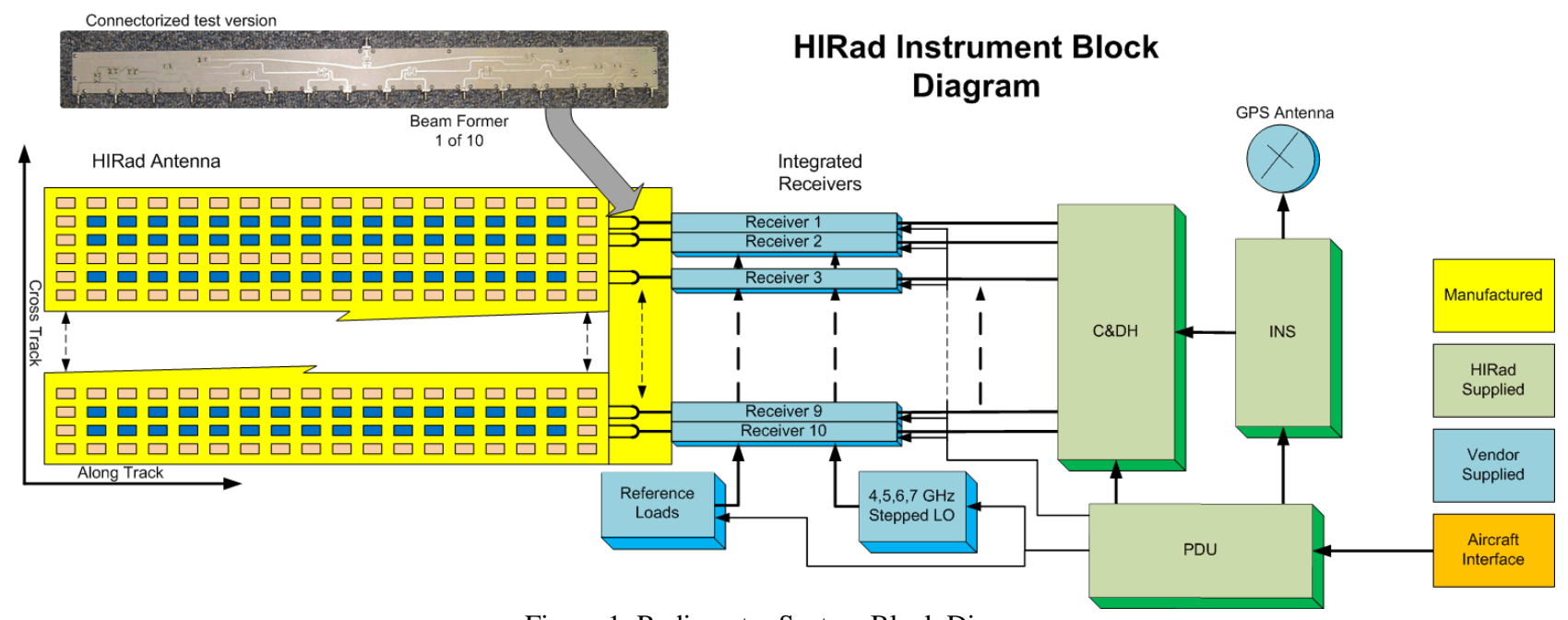

Figure 1: Radiometer System Block Diagram

\section{TEST PROCEDURE}

Tests to measure the element patterns, and the cross-track and along-track interference patterns, in the HIRAD system were conducted in an anechoic chamber using a broadband noise diode as a transmitting source. In addition, these scans were repeated with the array rotated $90^{\circ}$ in order to get a measurement of both the co-pol and cross-pol patterns. The scans were also done with the noise diode off, and these background readings were subtracted from the scans with the source on to remove chamber wall contributions.

A "G-Matrix" is constructed by sampling the visibility function at all possible spacings of antenna pairs, plus the zeroth spacing (element pattern) [4,5]. Inverting the GMatrix, (described in the next section), produces reconstructions of brightness temperature images. The instrument's $36(N)$ baselines assign $M(=2 N+1)$ visibility samples at 4 stepped frequencies giving 292 visibility samples for each scan.

The test data underwent initial performance testing to verify that each baseline was producing visibility samples with the expected spatial frequency response. The principal plane cross-scan produces interference patterns as the cross-correlation of each antenna pair according to the relation

$$
\operatorname{real}\left(S_{1} \times S_{2}^{*}\right)=\cos ^{2}(\theta) \times \cos \left(2 \pi D_{x} x\right)
$$

where

$$
x=\sin (\theta)
$$

$$
D_{x}=n \frac{\lambda}{2}, \lambda=0,1,2 \ldots 36
$$

and the complex cross-correlation is computed as $[2,4,5]$

$$
S_{1} \times S_{2}{ }^{*}=\left(I_{1} I_{2}+Q_{1} Q_{2}\right)+j\left(I_{2} Q_{1}-I_{1} Q_{2}\right) .
$$

Due to this relation, the Fourier transform of the interference patterns has a peak at $D_{x}$, the physical distance between the antennas, and this can be used to verify that the cross-correlations are producing interference patterns at the desired spatial frequencies. This relationship is verified in Fig. 2, which plots the power spectrum peak position for all 36 baselines.

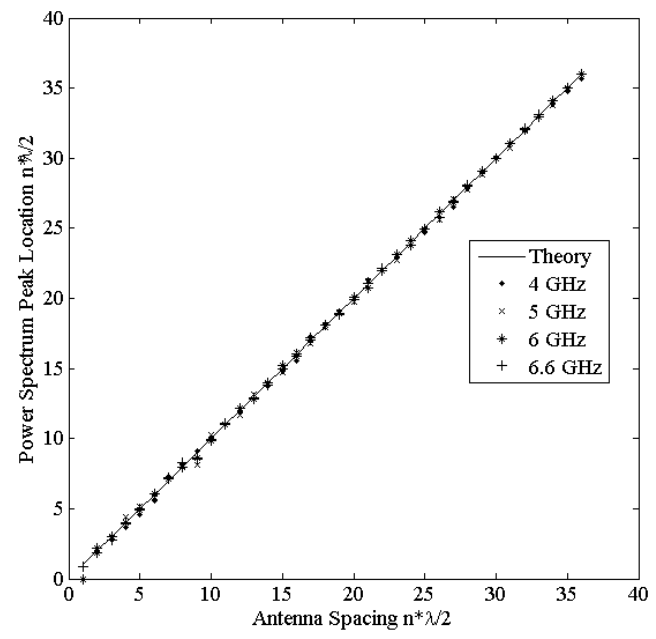

Figure 2: Power spectrum peak location for all 36 baselines, averaged over subbands $2-15$. 

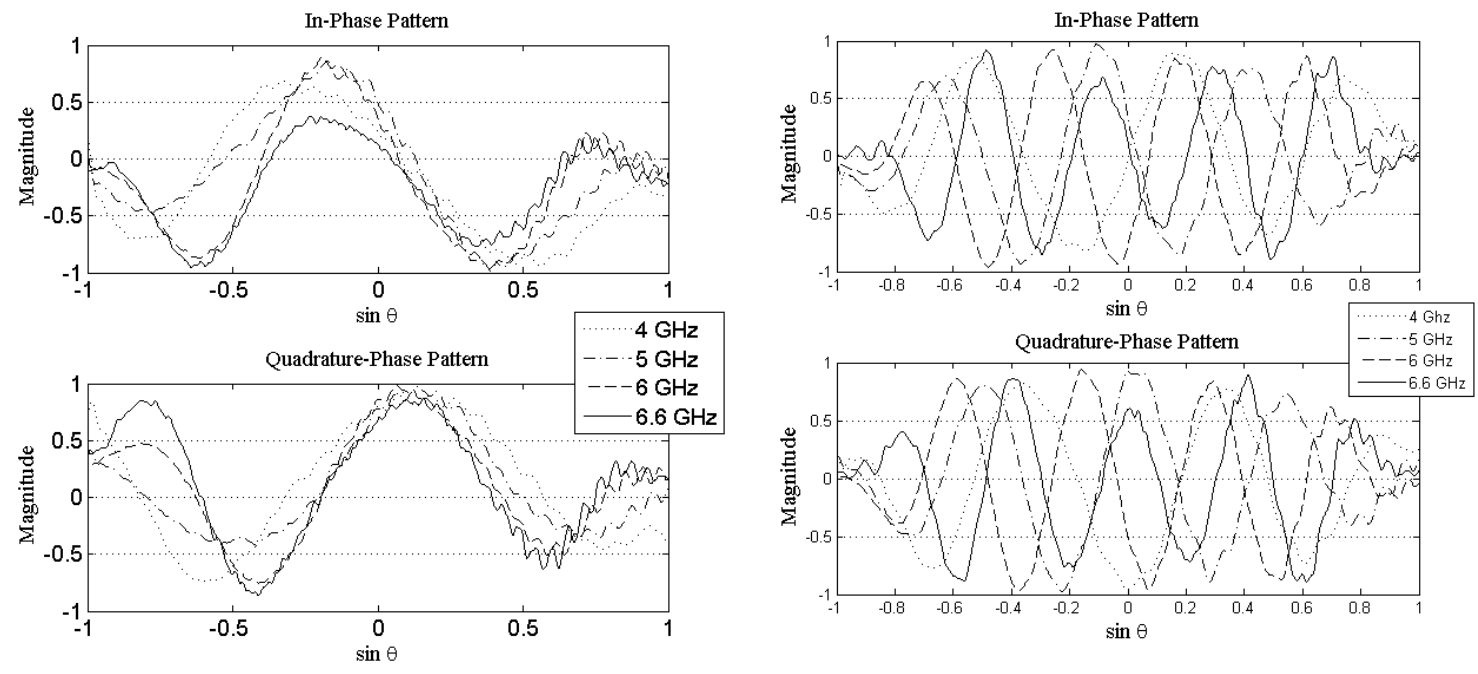

Figure 3: Interference Patterns for antenna pairs with baselines $2 * \lambda / 2$ and $5 * \lambda / 2$, respectively
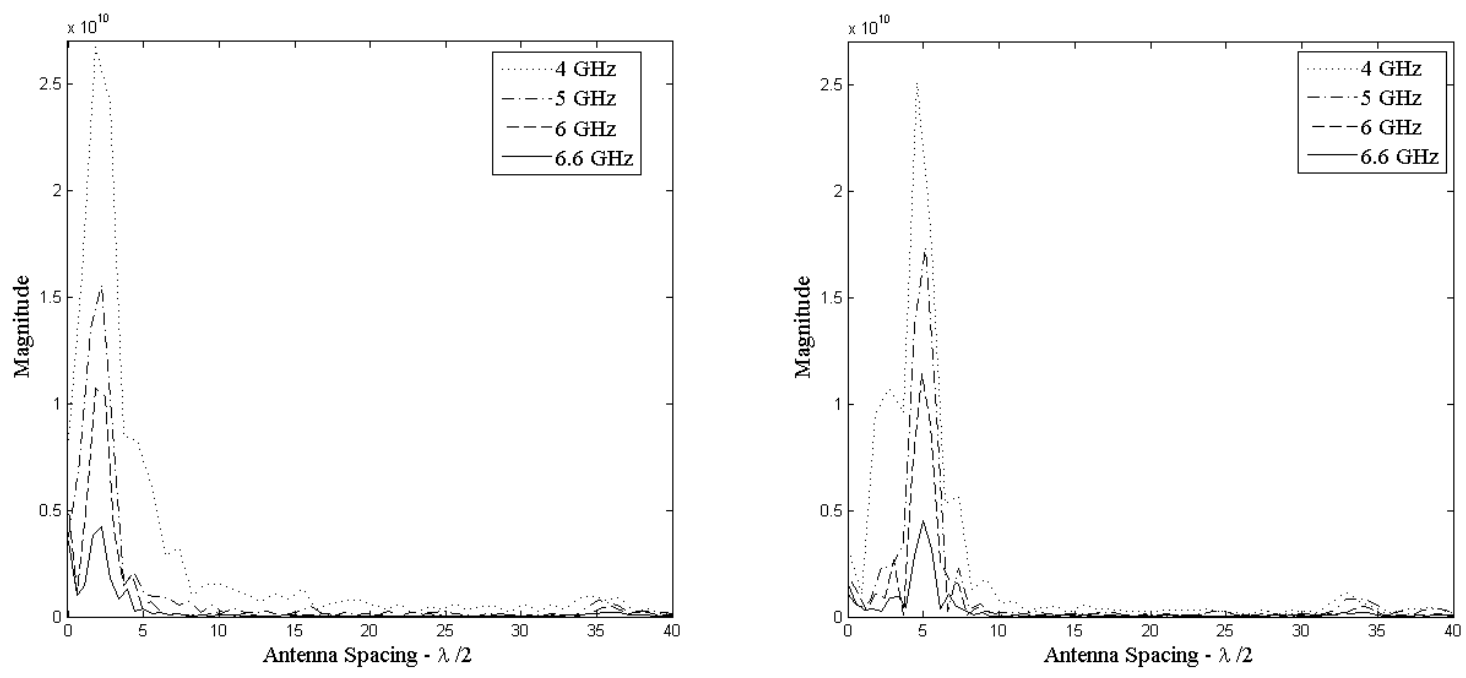

Figure 4: Power spectra for antenna pairs with baselines $2 * \lambda / 2$ and $5 * \lambda / 2$, respectively

Figs. 3 and 4 show examples at two HIRAD baselines of cross-correlations and their corresponding power spectra across all four frequency channels. The cross-correlations of antenna elements 1 and 3 (which have a spacing of $n=2$ ), and 8 and 9 (which have a spacing of $n=5$ ), can be seen in Fig. 3, with the power spectra in Fig. 4. The abscissa in Fig. 4 represents $n$ of equation (3). The peaks in these graphs are at approximately $n=2$ and $n=5$, as expected. Also noticeable in the figures are additional power contributions outside of the peak from the other antennas (near 36 in the $n=2$ figure and 34 in the $n=5$ ). These complex contributions are all factored into the Greconstruction $[4,5]$.

\section{IMAGE RECONSTRUCTION}

After acquiring the full complement of visibility samples from the anechoic chamber data, the G-Matrix can be constructed. The G-Matrix relates visibilities $(V)$ to the observed scene $\left(T_{B}\right)$ in the matrix representation of the forward model.

$$
V=G T_{B}
$$

Given the number of visibilities $M(=2 N+1)$ and the number of pixels in the observed scene, $P$, the G-Matrix is 
an MxP matrix. Its pseudo-inverse is operated on $V$ to reconstruct the brightness temperature according to $[4,5]$

$$
\hat{T}=G^{\prime} V
$$

where

$$
G^{\prime}=G^{t}\left(G G^{t}\right)^{-1}
$$

The Visibility samples such as those in Figure are sampled to fill the 73 rows of the G-Matrix and separate matrices are formed for each of the 16 subbands and each of the 4 stepped frequencies for a total of 64 G-Matrices. These will operate on the visibility function using equation (7) to reproduce a brightness image of the antenna scene.

\section{SUMMARY}

From the anechoic chamber tests, the full complement of visibility samples is available to populate the G-Matrix. All 36 baselines are available at subbands 2-15. Continuing work will be focused on analyzing the point response of the image reconstruction algorithm.

\section{ACKNOWLEDGEMENT}

The authors would like to thank S. Misra and S. Gross (University of Michigan) for their valuable assistance.

\section{REFERENCES}

[1] C.Ruf, R. Amarin, M.C. Bailey, B. Lim, R. Hood, M. James, J. Johnson, L. Jones, V. Rohwedder and K. Stephens, "The hurricane imaging radiometer - An octave bandwidth synthetic thinned array radiometer," Proc. IGARSS 2007, Barcelona, 23-27 July 2007.

[2] L. Jones, "An application for: Collaborative R\&D initiative for the Gulf of Mexico - Development of hurricane forecasting flight instrument HIRAD," Univ. Central Florida, Tech. Rep., 2009.

[3] C. S. Ruf "Numerical Annealing of Low-Redundacy Linear Arrays," IEEE Trans. Ant. And Prop, vol. 41, pp. 85-90, Jan.1993.

[4] C. S. Ruf, C. T. Swift, A. B. Tanner and D. M. Le Vine, "Interferometric Synthetic Aperture Microwave Radiometry for the Remote Sensing of the Earth," IEEE Trans. Geosci. Remote Sens., vol. 26, no. 5, pp. 597-611, Sep. 1988.

[5] A. B. Tanner and C. T. Swift, "Calibration of a Synthetic Aperture Radiometer," IEEE Trans. Geosci. Remote Sens., vol. 31, no. 1, pp. 257-267, Jan. 1993. 